\title{
FABRICAÇÃO DE PAINÉIS DE PARTÍCULAS DE MADEIRA TAUARI (Couratari oblongifolia) UTILIZANDO RESINA POLIURETANA DE MAMONA
}

\section{PRODUCTION OF PARTICLEBOARD PANELS FROM TAUARI (Couratari oblongifolia) WOOD RESIDUES USING CASTOR OIL POLYURETHANE RESIN}

\author{
Washington Luis França SANTOS ${ }^{1}$ \\ Antônio Jorge Parga da SILVA ${ }^{2}$ \\ Aluísio Alves CABRAL JÚNIOR ${ }^{3}$ \\ José Manuel Rivas MERCURY
}

\begin{abstract}
RESUMO
Neste trabalho foi estudada a fabricação de painéis de partículas (particleboard) de madeira tauari (Couratari oblongifolia) como forma de melhorar o aproveitamento da madeira oriunda do manejo extrativo da região amazônica, visando diminuir o impacto ambiental provocado pela retirada destas árvores. Os resíduos foram aglomerados com resina poliuretana de mamona, tipo bi componente. Os compósitos foram conformados com densidade nominal de $1000 \mathrm{~kg} \cdot \mathrm{m}^{-3}$ por prensagem uniaxial a uma pressão de $5 \mathrm{MPa}$, a 90,110 e $130^{\circ} \mathrm{C}$. Para a caracterização dos painéis foram realizados os ensaios de Densidade aparente $\left(D_{A P}\right)$, Teor de Umidade $(U)$, Absorção de água $(A A)$, Inchamento na espessura (IE), Flexão Estática (MOR e MOE), Tração perpendicular (TPP) e Arrancamento de parafuso (AP), segundo as recomendações da norma NBR 14810-3 da ABNT (2006b). Os resultados mostraram que os painéis fabricados com o resíduo da madeira tauari independentemente da temperatura de conformação apresentam densidade média entre 930 e $941 \mathrm{~kg} \cdot \mathrm{m}^{-3}$, com valores de tração perpendicular (ligação interna), inchamento na espessura superiores aos estabelecidos pelas normas NBR 14810-2 (ABNT, 2006a) e ANSI A208.1 (1999) sendo classificados de acordo como material de alta densidade recomendado para uso industrial e comercial.
\end{abstract}

Palavras chave: Painéis de partículas; Poliuretana de mamona; Propriedades físico-mecânicas; Madeira tauari.

The present paper examines the characteristics of wood wastes from Tauari (Couratari oblongifolia) trees used to produce particleboard panels agglomerated with castor oil resin (polyurethane) as a way to reduce the environmental impact of wood extraction in the amazon region. The composites were conformed with a target density of $1000 \mathrm{~kg}^{\circ} \mathrm{m}^{-3}$ by uniaxial pressing $(5 \mathrm{MPa})$ at 90,110 and $130^{\circ} \mathrm{C}$. The characterization of the panels was carried out by the following tests: Apparent density $\left(D_{A P}\right)$, Moisture $(U)$, Water Absorption (AA), thickness swelling (IE), Static bending strength: MOR (Modulus of rupture) and MOE (Modulus of elasticity), Tension perpendicular to the board surface (TPP) and Screw withdrawal (AP), following NBR 14810-3 of the Brazilian Technical Standards Association - ABNT (2006b). The results showed that panels produced from wood waste tauari regardless of the temperature of conformation have average density between 930 and $941 \mathrm{kgm}-3$, with values of tension perpendicular to the board surface (internal bond) and thickness swell higher than those defined by the standards NBR 14810-2 (ABNT, 2006a) and ANSI A208.1 (1999) and can be classified as high-density panels suitable for industrial and commercial use.

Keywords: Particleboard, Castor oil polyurethane, physical-mechanical properties, tauari wood.

\footnotetext{
${ }^{1}$ Desenhista Industrial, Professor M.Sc. em Engenharia e Ciências dos Materiais-Departamento de Desenho/DDE- Instituto Federal de Educação, Ciência e Tecnologia do Maranhão - Avenida Getúlio Vargas,04 - Monte Castelo CEP 65030-005 - São Luis - MA washington@ifma.edu.br

${ }^{2}$ Engenheiro Civil, Professor Dr. em Engenharia e Ciências dos Materiais-Departamento de Construção Civil/DCC- Instituto Federal de Educação, Ciências e Tecnologias do Maranhão - Avenida Getúlio Vargas,04 - Monte Castelo CEP $65030-005$ - São Luis - MA parga@ifma.edu.br

${ }^{3}$ Licenciado em Física, Doutor em Engenharia de Materiais, Professor do Programa de Pós-graduação em Engenharia dos Materiais (PPGEM), Departamento de Física (DCE) - Instituto Federal de Educação, Ciência e Tecnologia do Maranhão - Avenida Getúlio Vargas, 04 - Monte Castelo CEP 65030-005 - São Luis - MA - acabraljr@ifma.edu.br

${ }^{4}$ Engenheiro Químico, Professor Dr. em Engenharia e Ciências dos Materiais-Programa de pós graduação em Ciência e Engenharia dos Materiais/PPGEM - Instituto Federal de Educação, Ciências e Tecnologias do Maranhão - Avenida Getúlio Vargas,04 - Monte Castelo CEP 65030-005 - São Luis - MA - rivascefetma@gmail.com
} 


\section{INTRODUÇÃO}

O manejo florestal comunitário é uma prática de sustentabilidade que preza pela exploração racional dos recursos florestais para preservação das matas e ecossistemas. A utilização do manejo como ferramenta de preservação tem aumentado consideravelmente nos últimos anos na Amazônia (Amaral \& Amaral Neto, 2005). Esta prática se concentra, atualmente, na redução da exploração de madeira, sendo impulsionada pelas ONGs e governos locais por meio de incentivos e subsídios (Amaral \& Amaral Neto, 2005). A exploração seletiva de madeira e as queimadas causam drásticos impactos ambientais, principalmente a diminuição das espécies exploradas, o que afeta a regeneração natural das árvores (Monteiro et al., 2004; Oliveira et al., 2003).

Uma alternativa que pode contribuir para a redução do desperdício da madeira é o uso dos resíduos (folhas, galhos, costaneiras, aparas, cascas, serragem ou maravalhas) para a fabricação de materiais compósitos chamados de painéis de partículas aglomeradas (Chamma \& Leão, 2008; Silva, 2006; Silva \& Lahr, 2007, Poleto, 2007) como forma de reduzir os custos e aumentar o faturamento nos assentamentos rurais (Carvalho \& Oliveira, 2010), uma vez que estes resíduos representam $50,7 \%$ do total das toras produzidas (Brito, 1995).

O Tauari (Couratari oblongifolia) é uma planta pertencente à família Lecythidaceae, que ocorre em toda a região amazônica, principalmente nos estados do Pará, Amazonas, Acre, Rondônia e Maranhão (Vasconcelos et al., 2001). A madeira tauari possui características como: ser moderadamente pesada $\left(620 \mathrm{~kg} \cdot \mathrm{m}^{-3}\right)$ e macia ao corte; cerne e alburno indiferenciados quanto à cor, brancopalha, levemente rosado; textura média, grã direita; superfície ligeiramente lustrosa e lisa ao tato; cheiro e gosto imperceptíveis (Manieri, 1980), porém são quase inexistentes registros na literatura sobre a utilização desta madeira na confecção de chapas de partículas aglomeradas.

De acordo com Maloney (1977), a temperatura de processamento é uma das propriedades mais significativas na fabricação de painéis de partículas aglomeradas. Adicionalmente os seguintes fatores devem ser considerados: espécie de madeira, tamanho e geometria das partículas, pressão de compactação, tipo de resina e/ou adesivo e seu tempo de mistura.

$\mathrm{Na}$ literatura, vários trabalhos sobre fabricação de painéis relatam a utilização de fibras ou particulas de diferentes fontes lignocelulosicas e agentes aglutinantes sintéticos ou adesivos para a união (Leão et al., 1998; Iwakiri et al., 2002; Carneiro et al., 2004; Iwakiri et al., 2004, Bonelli et al., 2005; Fonseca, 2005; Poleto, 2007; Que et al., 2007; Buyuksari et al., 2010) das partículas muitas vezes por ação de calor (Iwakiri et al., 2005). Entretanto o adesivo de poliuretana (Araújo, 1992), produzido a partir da reação do óleo de rícino (óleo de mamona) e o isocianato vem sendo muito utilizado devido ao fato do mesmo ser renovável, biodegradável e apresentar baixo grau de toxicidade (König et al., 1999; Claro Neto, 1997; Ogunniyi, 2005).

Neste trabalho, estuda-se viabilidade de produção de painéis de partículas fabricados com resíduos de madeira tauari aglomeradas com resina poliuretana de mamona e a influência da temperatura de compactação nas propriedades físico-mecânicas.

\section{MATERIAIS E MÉTODOS}

\section{Materiais}

Resíduos na forma de cavacos e flocos da madeira Tauari (Couratari oblongifolia) fornecidos pela indústria moveleira do município de João Lisboa (Maranhão, Brasil) foram recebidos em laboratório e secos a umidade constante de $5 \%$ em b.s. Os resíduos foram triturados em moinho de rotor vertical, com facas móveis e fixas (modelo MA 680 Marconi), até a obtenção de uma amostra homogênea. Após moagem, as partículas foram peneiradas em malhas $14-18$, sendo utilizado o material retido na malha 18 (1 mm - ABNT) para a confecção dos painéis.

Para aglomeração das partículas de madeira utilizou-se resina de poliuretana a base do óleo de mamona tipo bi componente (RPM) com densidade entre $0,9-1,2 \mathrm{~g} / \mathrm{cm}^{3}$, fabricada pela $\mathrm{KEHL} \mathrm{In-}$ dústria e Comércio, São Carlos-SP, com $0,1 \%$ de formol livre após $24 \mathrm{~h}$.

\section{Preparação dos Compósitos}

$\mathrm{Na}$ preparação dos compósitos foram utilizados $1500 \mathrm{~g}$ do resíduo de madeira $(86,20 \%$ em peso) e $240 \mathrm{~g}$ da resina $(13,80 \%$ em peso) num traço de 1:2 sendo uma parte de pré-polímero, à base de di-isocianato, e duas partes de poliol. A resina foi adicionada às partículas e processada em um misturador durante cinco minutos. Após a homogeneização, a mistura foi conformada em uma prensa hidráulica com capacidade de 50 toneladas e aquecimento controlado até $200^{\circ} \mathrm{C}$ (modelo MA098/50 - Marconi) por 10 minutos nas temperaturas de 90,110 e $130^{\circ} \mathrm{C}$, a uma pressão de $5 \mathrm{MPa}$ e densidade nominal de $1000 \mathrm{~kg} / \mathrm{m}^{3}$ em um molde de $400 \times 400 \times 10 \mathrm{~mm}$. Foram confeccionados 4 painéis por tratamento no Laboratório de Madeiras e Estruturas de Madeira da USP - São Carlos - SP (LaMEM).

A Tabela 1 apresenta as condições experimentais utilizadas para a confecção dos painéis em laboratório e a nomenclatura utilizada para identificação de cada um dos compósitos.

\section{Caracterização físico-mecânica dos compósitos}

Os painéis produzidos foram mantidos a temperatura ambiente durante $48 \mathrm{~h}$. Destes painéis 
foram retirados aleatoriamente 12 corpos-de-prova (CP) por tratamento para cada ensaio físicomecânico: i) Para os ensaios de Densidade $\left(D_{A P}\right)$ e Tração Perpendicular (TPP) foram utilizados CP de dimensões $50 \times 50 \times 12 \mathrm{~mm}$; ii) Para os ensaios de Inchamento na espessura (IE) e Absorção de Água (AA) após $24 \mathrm{~h}, \mathrm{CP}$ de dimensões $25 \times 25 \times 12 \mathrm{~mm}$, foram usados; iii) Os ensaios de flexão estática (MOR e MOE), foram realizados em CP de 250 x 50 $x$ 12. iv) No ensaio de arranque de parafuso (AP) foram utilizados em CP de $250 \times 50 \times 24 \mathrm{~mm}$. Todos os ensaios foram realizados seguindo as recomendações da norma NBR 14810-3 da ABNT (ABNT, 2006b)

\section{Análise Estatística}

Para o tratamento dos dados as variáveis foram analisadas quanto a sua normalidade pelo teste de Shapiro-Wilk, utilizando nível de significância 0,05. Foi utilizada a Análise de Variância (ANOVA One-Way) seguido do post-hoc Tukey para comparação das variáveis que apresentaram distribuição normal. As variáveis: arranque parafu-
SANTOS, W. L. F. et al. Fabricação de painéis...

so, absorção de água e MOR foram comparadas pelo teste Kruskall-Wallis, adotando nível de significância de 0,05.

\section{RESULTADOS E DISCUSSÃO}

\section{Caracterização física dos painéis}

A determinação das propriedades físicomecânicas, tais como ligação interna, flexão estática, resistência ao arranque de parafusos, densidade, absorção de água e inchamento na espessura, são um indicativo da qualidade dos painéis (Iwakiri et al., 2005). Na Figura 1 apresentam-se os valores da densidade dos painéis estudados. Observa-se que os valores médios da densidade nas temperaturas de 90,110 e $130^{\circ} \mathrm{C}$ correspondem a 930,3, 932,2 e $941,8 \mathrm{~kg} / \mathrm{m}^{3}$ respectivamente, com coeficientes de variação não superiores a $4 \%$. A densidade dos painéis foram estatisticamente semelhantes, uma vez que a análise de variância não revelou diferença significativa $(F=0,4813, p>0,05)$.

\begin{tabular}{|c|c|c|c|c|c|c|}
\hline \multicolumn{6}{|l|}{ Tabela 1. Organização dos ensaios } \\
\hline Tratamento & $\mathrm{T}\left({ }^{\circ} \mathrm{C}\right)$ & Código & Painéis & $\begin{array}{c}\text { Pressão } \\
(\mathrm{MPa})\end{array}$ & $\begin{array}{c}\text { Densidade } \\
\left(\mathrm{kg} / \mathrm{m}^{3}\right)\end{array}$ & Aglomerante \\
\hline 1 & 90 & MM90 & $\mathbf{0 4}$ & 5 & 1000 & RPM $^{*}$ \\
\hline 2 & 110 & MM110 & 04 & 5 & 1000 & RPM \\
\hline 3 & 130 & MM 130 & 04 & 5 & 1000 & RPM \\
\hline
\end{tabular}

\begin{tabular}{|c|c|c|c|c|}
\hline \multirow{2}{*}{ Tratamento } & \multicolumn{4}{|c|}{ Medias \pm Erro padrão } \\
\hline & MOR (MPa) & MOE (MPa) & TPP (MPa) & $\mathrm{AP}(\mathrm{N})$ \\
\hline MM90 & $15,85 \pm 0,25 a$ & $1336,1 \pm 34,43 \mathbf{a}$ & $1,55 \pm 0,04 \mathbf{a}$ & $1668,9 \pm 19,29 a$ \\
\hline MM110 & $16,02 \pm 0,47 \mathbf{a}$ & $2011,7 \pm 53,04$ b & $1,56 \pm 0,09 \mathbf{a}$ & $2098,8 \pm 236,3 \mathbf{a}$ \\
\hline MM130 & $19,58 \pm 0,57 \mathbf{b}$ & $2378,5 \pm 89,86$ c & $1,70 \pm 0,07 \mathbf{a}$ & $1719,1 \pm 16,67$ b \\
\hline
\end{tabular}

\begin{tabular}{|c|c|c|c|c|}
\hline \multicolumn{5}{|c|}{ Tabela 2b. Coeficientes de variação das propriedades mecânicas dos painéis. } \\
\hline \multirow{2}{*}{ Tratamentos } & MOR (MPa) & MOE (MPa) & TPP (MPa) & AP (N) \\
\cline { 2 - 5 } & $5.54 \%$ & $8.93 \%$ & $8.56 \%$ & $4.00 \%$ \\
\hline MM90 & $10.26 \%$ & $9.13 \%$ & $20.26 \%$ & $39.00 \%$ \\
\hline MM110 & $10.17 \%$ & $13.09 \%$ & $14.16 \%$ & $2.12 \%$ \\
\hline
\end{tabular}


SANTOS, W. L. F. et al. Fabricação de painéis...

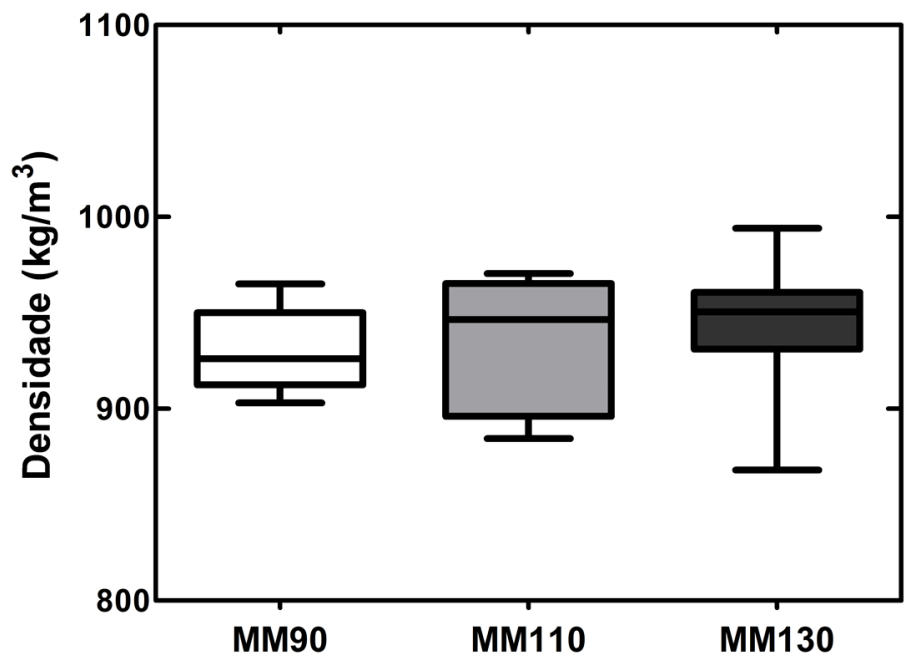

Figura 1. Densidade média dos painéis de madeira tauari.

Estes valores são inferiores à densidade nominal de $1000 \mathrm{~kg} / \mathrm{m}^{3}$ pré-estabelecida, porém semelhantes aos obtidos por Sartori et al. (2012) e Fiorelli et al. (2011) que estudaram painéis à base de bagaço da cana-de-açúcar aglomerados com poliuretana bicomponente à base de óleo de mamona. Os valores de densidade obtidos permitem classificá-los como painéis de alta densidade de acordo com a Norma ANSI A208.1-1999 (1999). Por outro lado, a diferença observada entre a densidade nominal e a densidade média dos painéis já foi reportada por outros autores (Contreras et al.,
2006; Moreno et al., 2007), e atribuída à perda de matéria prima (partículas de madeira e resina) durante o processo de mistura e prensagem manual.

A umidade média dos compósitos fabricados variou entre 5,51 e $8,29 \%$ (Figura 2), valores que se situam no intervalo de 5-11\%, como recomendado pela norma NBR 14810-2 (ABNT, 2006a), sendo inferiores aos valores mínimos de 8 $\% \pm 2$, com relação às partículas secas, recomendados por Deppe \& Erns, citados por Moslemi (1974).

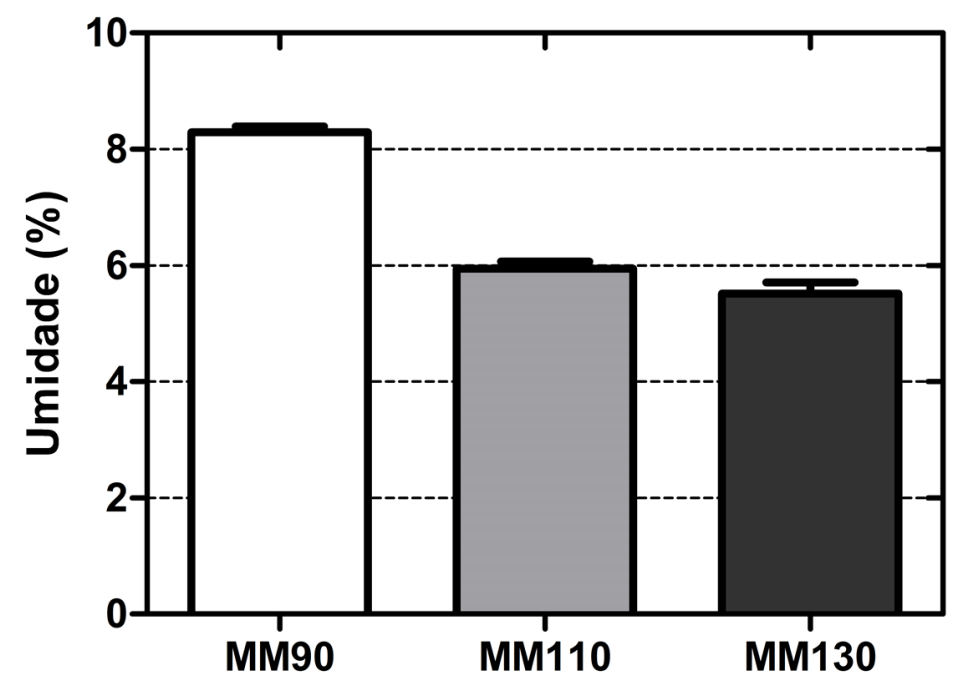

Figura 2. Variação da umidade média dos painéis de madeira tauari.

Os tratamentos feitos a 110 e $130^{\circ} \mathrm{C}$ apresentaram valores de umidade significativamente menores que os observados nos painéis tratados a $90^{\circ} \mathrm{C}(\mathrm{F}=109.8, \mathrm{p}<0.001)$. A diferença observada pode ser explicada pelo aumento da temperatura de prensagem responsável pela evaporação da água aderida à superfície das partículas durante o processamento que provoca também a cura da resina com uma melhor densificação do painel (Riegel, 2008).
Na Figura 3, são apresentados os resultados do ensaio de AA após $24 \mathrm{~h}$ dos materiais estudados. Pode-se observar que, o valor médio da AA dos painéis obtidos à temperatura de $90^{\circ} \mathrm{C}$, apresenta valores superiores a $30 \%$. Com o aumento da temperatura dos painéis para 110 e $130^{\circ} \mathrm{C}$, a AA diminui significativamente (de 22,49 a $19,87 \%$ ), sendo esta diferença estatisticamente significativa $(F=56,6 ; p<0.001)$. 
SANTOS, W. L. F. et al. Fabricação de painéis...

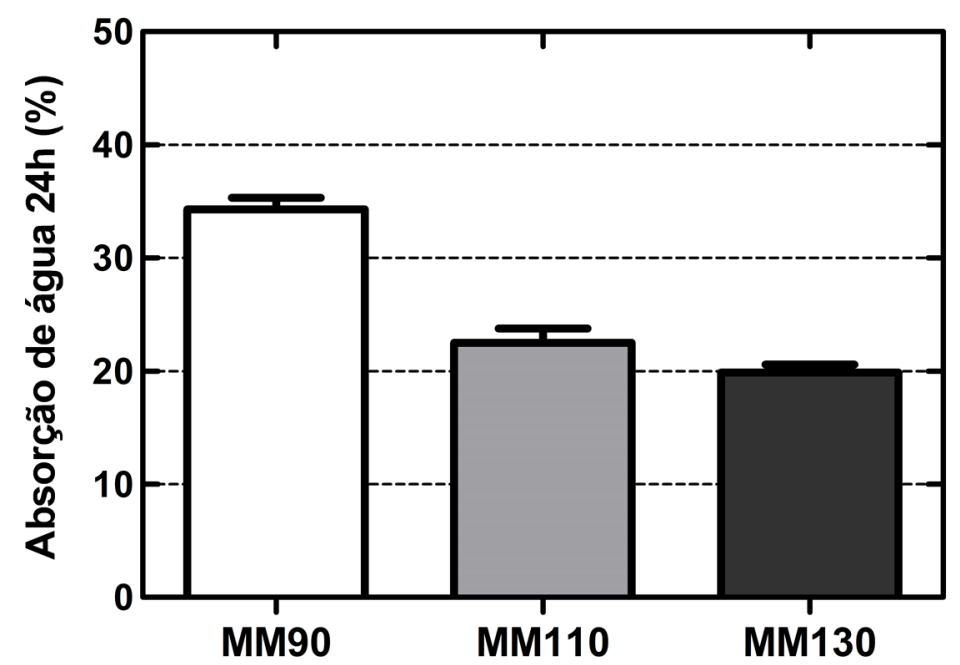

Figura 3. Absorção de água após $24 \mathrm{~h}$ dos painéis de madeira tauari.

A diferença observada nos valores médios da AA dos compósitos está relacionada com a diminuição da viscosidade da resina. O aumento da temperatura de processamento melhora a impregnação das partículas o que leva a diminuição da espessura dos painéis durante a compactação e consequentemente aumenta o grau de polimerização da resina utilizada.

Valores baixos de AA estão associados a elevadas densidades dos painéis e a elevada relação de compressibilidade $(1,61: 1)$. É importante observar que a norma NBR 14810-2 (ABNT, 2006a) não apresenta exigências quanto à $A A$ de aglomerados de partículas. Os valores médios de AA dos painéis tratados a 110 e $130{ }^{\circ} \mathrm{C}$ indicam que estas temperaturas são adequadas para a obtenção de painéis de madeira tauari de alta densidade (ANSI,1999). Estes resultados estão de acordo com aqueles encontrados por Fiorelli et al. (2012) para painéis fabricados com fibra de coco e resina poliuretana de mamona.

Os valores do IE após $24 \mathrm{~h}$ de imersão em água estão representados na Figura 4. Cabe lembrar que a norma NBR 14810-2 (ABNT, 2006a) não estabelece valores de IE para chapas aglomeradas. Entretanto este ensaio permite observar com maior precisão as diferenças entre os tratamentos, bem como as condições de adesão e de resistência das partículas do painel após $24 \mathrm{~h}$ de imersão em água.

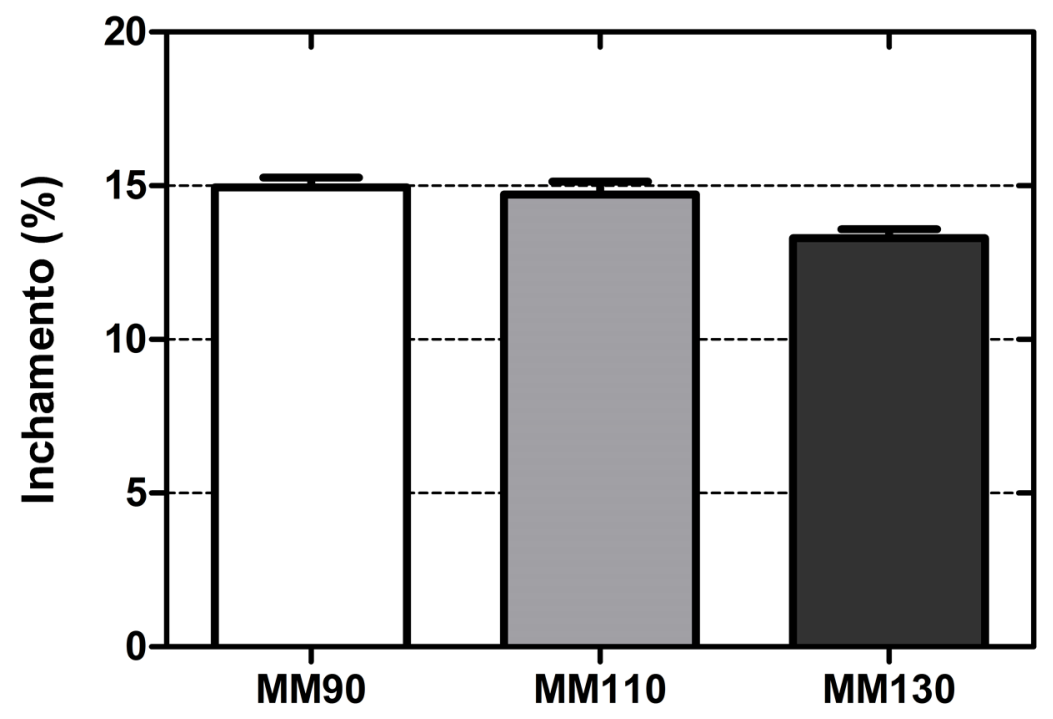

Figura 4. Inchamento na espessura após 24 h dos painéis de madeira tauari 
Pode-se observar (Figura 4) que os valores médios de IE após 24 h são inferiores a $15 \%$. Os painéis tratados a $130^{\circ} \mathrm{C}$ apresentaram IE significativamente menor que os realizados na temperatura de 90 e $110^{\circ} \mathrm{C}(\mathrm{F}=6,47, \mathrm{p}<0.05)$, o que indica que a temperatura de prensagem foi uma variável preponderante na cura do adesivo utilizado. Entretanto, os valores médios obtidos para IE em todos os painéis foram inferiores aos obtidos por Sartori et al. (2012) e Fiorelli et al. (2011).

\section{Caracterização mecânica dos painéis}

$\mathrm{Na}$ Tabela 2(a-b) encontram-se os resultados dos ensaios mecânicos de resistência à flexão estática (MOR e MOE), tração perpendicular (TPP) e arrancamento de parafuso (AP) e seus respectivos coeficientes de variação. É possível observar na Tabela 2-a que os valores do MOR aumentam com o aumento da temperatura de processamento. À temperatura de $130^{\circ} \mathrm{C}$ o valor médio do MOR $(19,57 \mathrm{MPa})$ é superior àquele recomendado pela norma NBR 14810-2 (ABNT, 2006a), sendo identificada diferença estatística significativa entre este e os dois primeiros valores. Os resultados mostram que os painéis fabricados a $130^{\circ} \mathrm{C}$ mediante a utilização de resina bicomponente à base de óleo de mamona e partículas de madeira tauari apresentam potencial para a fabricação de painéis de partículas que podem ser classificados como painéis de alta densidade indicados para uso comercial e industrial.

Em nenhum dos tratamentos realizados, os valores médios do módulo de elasticidade (MOE) dos painéis (Tabela 2-a) atingiram o valor mínimo (2750 $\mathrm{MPa}$ ) recomendado pela norma A208-1-1999 (ANSI, 1999), sendo obtido após tratamento a $130^{\circ} \mathrm{C}(2378,5 \mathrm{MPa})$ um valor $15,62 \%$ inferior ao recomendado. Baixos valores de MOE já foram reportados por vários pesquisadores (Paes et al., 2011; Dias et al., 2008; Contreras et al., 2006; Iwakiri et al., 2004), os quais atribuíram este comportamento à má distribuição do adesivo, durante o processo de conformação dos painéis. Os ensaios de resistência na flexão estática apresentaram diferenças estatísticas entre os módulos de ruptura (MOR) e de elasticidade (MOE) conforme a variação da temperatura.
Já os resultados dos ensaios de TPP apresentados na Tabela 2-a, demonstram que, independente da temperatura de processamento, os valores médios desta propriedade variam entre 1,55 e 1,70 MPa, os quais são superiores àqueles recomendados pelas normas NBR 14810-2 (ABNT, 2006a), e A208.1:1999 (ANSI, 1999). Os valores médios de TPP não apresentaram diferenças significativas em nível de $5 \%$ de probabilidade.

Os valores médios de AP dos painéis produzidos neste estudo em todas as temperaturas estudadas são superiores ao mínimo de $1020 \mathrm{~N}$ para superfície e de $800 \mathrm{~N}$ para o topo, estabelecidos pela norma NBR 14810-2 (ABNT; 2006a). Os tratamentos a 90 e $110^{\circ} \mathrm{C}$ não apresentaram diferenças estatísticas significativas em nível de 5\%.

É importante ressaltar que os coeficientes de variação das propriedades de MOR, MOE e IB ficaram abaixo dos $20 \%$ recomendados, sendo estes valores uma garantia da consistência do processo de fabricação.

\section{CONCLUSÕES}

É possível produzir, em laboratório, painéis de partículas de madeira tauari aglomerados com resina poliuretana à base de óleo de mamona, com valores médios de densidade variando entre 930 e $940 \mathrm{~kg} \cdot \mathrm{m}^{-3}$.

Independente da temperatura utilizada, as chapas produzidas apresentaram valores de tração perpendicular às fibras (ligação interna) e arrancamento de parafuso superiores às exigências da norma brasileira e da comercial americana.

Os painéis conformados a $130^{\circ} \mathrm{C}$ apresentaram as melhores propriedades físicas e mecânicas entre os materiais compósitos fabricadas indicando ser esta a melhor temperatura de processamento.

\section{AGRADECIMENTOS}

Ao LaMEM, Laboratório de Madeiras e Estruturas de Madeira da USP - São Carlos que possibilitou a confecção dos painéis e a Fundação de Amparo à Pesquisa e ao Desenvolvimento Cientifico e Tecnológico do Estado do Maranhão (FAPEMA), pelo apoio financeiro.

\section{REFERÊNCIAS}

1. AMARAL, P.; AMARAL NETO, M. Manejo florestal comunitário: processos e aprendizagens na Amazônia brasileira e na América Latina. Belém: IEB;IMAZON, 2005. 44p.

2. AMERICAN NATIONAL STANDARDS INSTITUTE - ANSI. ANSI A208.1: Particleboard: specification. Gaithersburg, 1999.

3. ARAÚJO L. C. R. Caracterização Química, Térmica e Mecânica de Poliuretanas Elastoméricas Baseadas em Materiais Oleoquímicos. Dissertação de Mestrado, Universidade de São Paulo, Brasil (1992).

4. ASSOCIAÇÃO BRASILEIRA DE NORMAS TÉCNICAS - ABNT. NBR 14810-2. Chapas de madeira aglomerada. Parte 2: Requisitos. Rio de Janeiro, 2006a. 
5. ASSOCIAÇÃO BRASILEIRA DE NORMAS TÉCNICAS - ABNT. NBR 14810-3. Chapas de madeira aglomerada. Parte 3: Métodos de ensaio. Rio de Janeiro, 2006b. 6. BONELLI, C.M.C et al.. Comportamento térmico, mecânico e morfológico de compósitos de polietileno de alta densidade reciclado com fibra de piaçava. Polímeros, v. 15. n. 4, p. 256-260, 2005.

7. BRITO, E. O. Estimativa da produção de resíduos da Indústria brasileira de serraria e laminação de madeira. Revista da Madeira, v. 4, n. 26, p.34-39, 1995.

8. BUYUKSARI, U. et al. Evaluation of the physical, mechanical properties and formaldehyde emission of particleboard manufactured from waste stone pine Pinus pinea L. cones. Bioresource Technology, v.101, n. 1, p.255-259, 2010.

9. CARNEIRO, A. C. O. et al. Propriedades de chapas de flocos fabricadas com adesivo de uréia-formaldeído e de taninos da casca de Eucalyptus grandis W. Hill ex Maiden ou de Eucalyptus pellita F. Muell. Revista Ánvore, v. 28, n. 5, p. 715-724, 2004. 10. CARVALHO, R. da S.; OLIVEIRA, A. D. de. Viabilidade Econômica do Manejo Florestal Madeireiro em Projetos de Assentamento Extrativistas no Sudoeste da Amazônia. Cerne, v. 16, n. 4, p. 505-516, out./dez. 2010.

11. CHAMMA P.V.C., LEÃO A. L. Aproveitamento de resíduos sólidos na produção de painéis para aplicações arquitetônicas. Revista Energia na Agricultura, v. 23, n. 2, p. 73-84, 2008.

12. CLARO NETO, S. Caracterizações físico-químicas de um poliuretano derivado de óleo de mamona utilizado para implantes ósseos. 1997. 127 f. Tese (Doutorado) - Programa de pós-graduação em Química Analítica, Instituto de Química de São Carlos, Universidade de São Paulo, São Carlos, SP.

13. CONTRERAS, W. M. et al. Diseño de tableros de partículas de caña brava y adhesivo fenol - formaldehído. Revista Forestal Latinoamericana, v. 21, n. 39, p. 39- 55, 2006.

14. DIAS, F. M.; et al. Aplicação de resina poliuretana à base de mamona na fabricação de painéis de madeira aglomerada. In: Produtos derivados de madeira: síntese dos trabalhos desenvolvidos no Laboratório de Madeira e Estruturas de Madeira, SET-EESC-USP, p73-92, São Carlos, São Paulo, 2008.

15. FIORELLI, J. et al. Painéis de partículas à base de bagaço de cana e resina de mamona - produção e propriedades. Acta Scientiarum. Technology, v. 33, n. 4, p. 401-406, 2011.

16. FONSECA, F.M.C. Desenvolvimento e Caracterização de Compósitos à base Polietileno de Alta Densidade (PEAD) Reciclado com Fibras Vegetais. 2005, p.133. Dissertação (Mestrado), Redemat, Universidade Federal de Ouro Preto, UEMG, Belo Horizonte.

17. IWAKIRI, S. et al. Produção de compensados de Pinus taeda L.E e Pinus oocarpa Schiede com diferentes formulações de adesivo ureia formaldeído. Revista Árvore, v.26, n.3, p.371-375, 2002.

18. IWAKIRI, S. et al. Produção de painéis de madeira aglomerada de Grevillea robusta A. Cunn. ex R. Br.. Revista Árvore, v. 28, n.6, p. 883-887, 2004.

19. IWAKIRI, S. et al. Produção de painéis de madeira aglomerada de alta densificação com diferentes tipos de resinas. Scientia Forestalis, n. 68, p.39-43, ago. 2005.

20. KÖNIG JÚNIOR, B. et al. Biocompatibility of the polyurethane resin of the castor bean inserted into the alveolar bone of the dog., Annals of Anatomy, v.181, n. 6, p.582-584, 1999.

21. LEÃO, A. L. et al. Curaua fiber - A tropical natural fibers from Amazon - Potential and Application in Composites", In: International Conference on Advanced Composites, Hurghada, Egito, 1998. p. 557-564.

22. MALONEY, T. M. Modern particleboard \& dry-process fiberboard manufacturing. San Francisco, Miller Freeman, USA, 1977. $681 \mathrm{p}$

23. MANIERI, C. Fichas Características das Madeiras Brasileiras. $2^{a}$ ed. São Paulo: IPI, 1912,1980. 418p.

24. MONTEIRO et al. Impactos da Exploração Madeireira e do Fogo em Florestas de Transição da Amazônia Legal. Scientia Forestalis, n.65, p.11-21, 2004.

25. MORENO, P. A. P. at al. Utilización de bambusa vulgaris como una alternativa en la fabricación de tableros aglomerados de particulas. Revista Forestal Latinoamericana, v. 22, n. 42, p. 31-50, 2007.

26. MOSLEMI, A.A. Particleboard: Materials. Illinois: Southern Illinois University Press, 1974. v.1. 245 p.

27. OGUNNIYI, D.S. Castor oil: A vital industrial raw material. Review Paper In: Bioresource Technology, v. 97, n. 9, p.10861091, 2005.

28. OLIVEIRA, L.C. et al. Impactos da Exploração Seletiva de Madeira em Áreas em Processo de Fragmentação Florestal na Amazônia Ocidental. Cerne, v.9, n. 2, p. 213-220, 2003.

29. PAES, J. B. et al. Qualidade de chapas de partículas de Pinus elliottiii coladas com resina poliuretana sob diferentes combinações de pressão e temperatura. Ciência Florestal, v. 21, n. 3, p. 551-558, 2011.

30. POLETO, S.F.S. et al. Produção de Chapas de Partículas homogêneas (CPH) utilizando resíduos de espécies de reflorestamento. Reciclagem de resíduos para a construção civil. Belo Horizonte. FUMEC-FEA, 2007, v. 01, p 271-292.

31. QUE, Z. et al. Effects of urea-formaldehyde resin mole ratio on the properties of particleboard. Building and Environment, v. 42, n. 3, p.1257-1263, 2007.

32. RIEGEL, I. et al. Análise Termogravimétrica da Pirólise da Acácia-Negra (Acacia mearnsii) Cultivada no Rio Grande do Sul, Brasil. Revista Árvore, v.32, n.3, p.533-543, 2008.

33. SARTORI D.L. et al. Painel em Madeira de Reflorestamento e Chapas de Partículas para Instalações Rurais. Floresta e Ambiente, v. $19, \mathrm{n} 2$, p. 171-178, 2012.

34. SILVA, A. J. P. Aplicação de partículas longas e orientadas de bagaço de cana de açúcar na produção de painel particulado similar ao OSB. 2006. 144 f. Tese (Doutorado) Programa de Pós-Graduação em Ciência e Engenharia de Materiais. Interunidades. Universidade de São Paulo, Escola de Engenharia de São Carlos (USP-EESC), São Carlos, SP.

35. SILVA, A. M. da e LAHR, F. A. R. Chapas de partículas Confeccionadas com Resíduos de madeiras tropicais de baixa e média densidade. Reciclagem de resíduos para a construção civil. Belo Horizonte. FUMEC-FEA, 2007, v. 01, p 343-365.

36. VASCONCELOS, F. J. et. al. Madeiras tropicais de uso industrial do Maranhão: Características tecnológicas. Manaus, Amazonas: INPA, Coordenação de Pesquisas em Produtos Florestais: UFMA, Centro Tecnológico, 2001, 96 p.

Recebido em 05/05/2011

Aceito em 05/08/2013 